\title{
Relay-Assisted Free-Space Optical Communication
}

\author{
Majid Safari, Student Member, IEEE, and Murat Uysal, Senior Member, IEEE
}

\begin{abstract}
In this paper, we present relay-assisted transmission as a powerful fading mitigation tool for free-space optical systems operating in atmospheric turbulence channels. We study both serial (i.e., multi-hop transmission) and parallel (i.e., cooperative diversity) relaying encoupled with amplify-and-forward and decode-and-forward modes. We consider an aggregated channel model which takes into account both path-loss and turbulence-induced log-normal fading. Since fading variance is distance-dependent in free-space optical systems, relay-assisted transmission takes advantage of the resulting shorter hops and yields significant performance improvements. We derive outage probability of the relaying schemes under consideration which are further confirmed through Monte-Carlo simulations. Our outage probability analysis demonstrates that an impressive performance improvement of $18.5 \mathrm{~dB}$ is possible with the use of a single relay at a target outage probability of $10^{-6}$.
\end{abstract}

Index Terms-Free-space optical systems, cooperative diversity, fading channels.

\section{INTRODUCTION}

$\mathbf{F}$ REE-SPACE optical (FSO) communication refers to terrestrial line-of-sight optical transmission through the atmosphere. This technology has recently attracted a renewed interest within the research community although its roots can be traced back to Alexander Graham Bell's "photophone" [1]. In this first FSO experiment carried out on February 18, 1880, Bell was able to transmit voice signals through a modulated beam of light via atmosphere for a distance of about $200 \mathrm{~m}$. Although Bell's photophone never came out as a commercial product, it has successfully demonstrated the potential of FSO transmission.

Today's FSO systems use either lasers or LEDs (light emitting diodes) to transmit a modulated beam of visible/infrared light [2]. These systems are license-free with high-bandwidth capacity providing a cost-effective and easy-to-install alternative to fiber optics. They further provide an inherent security due to the nature of their directional and narrow beams which make eavesdropping and jamming nearly impossible. With its unique features, FSO communication is appealing for a number of applications including last-mile access, fiber backup, back-haul for wireless cellular networks, and disaster recovery [3].

Manuscript received December 1, 2007; revised March 2, 2007; accepted March 31, 2007. The associate editor coordinating the review of this paper and approving it for publication was N. Arumugam. This paper was presented in part at the Asilomar Conference on Signals, Systems, and Computers, Monterey, CA, USA, November 2007.

The work of M. Uysal is supported in part by a Natural Sciences and Engineering Research Council of Canada (NSERC) Special Research Opportunity Grant (SROPJ305821-05).

The authors are with the Department of Electrical and Computer Engineering, University of Waterloo, Waterloo, ON, N2L3G1, Canada (e-mail: m3safari@uwaterloo.ca,muysal@ece.uwaterloo.ca).

Digital Object Identifier 10.1109/T-WC.2008.071352
Despite the major advantages of FSO, its widespread use has been hampered by its rather disappointing performance for long-range links. For link ranges longer than $1 \mathrm{~km}$, atmospheric turbulence-induced fading becomes a major performance limiting factor in FSO systems [4]. A number of fadingmitigation techniques have been proposed in the literature including error-correcting codes [5], [6], maximum-likelihood sequence estimation [7], and spatial diversity [8]-[11]. Among those, spatial diversity is particularly attractive with its lower complexity. Spatial diversity involves the use of multiple transmit and/or receive apertures and has been extensively studied in the context of wireless radio-frequency (RF) communication before it was applied to FSO communication. In this paper, we borrow another well-studied concept - cooperative diversity from wireless RF literature to apply within the context of FSO communications.

Cooperative diversity has been recently introduced as an alternative way of realizing spatial diversity advantages [12][14]. The main idea behind cooperative diversity is based on the observation that in a wireless RF channel, the signal transmitted by the source node is overheard by other nodes, which can be defined as partners or relays. The source and its partners can jointly process and transmit their information, creating a virtual antenna array although each of them is equipped with only one antenna. Multihop transmission is an alternative relay-assisted transmission scheme which employs the relays in a serial configuration [15], [16]. Such schemes are typically used to broaden the signal coverage for limitedpower transmitters and do not offer performance improvement against fading effects in wireless RF environments, i.e., it does not increase the diversity order [12].

To the best of our knowledge, relay-assisted FSO transmission was first proposed by Acampora and Krishnamurthy in [17]. Their work, however, has a networking perspective and does not address the physical layer aspects which our paper aims to focus on. In [18], Akella et.al. have studied the bit error rate performance of a decode-and-forward FSO multi-hop scheme. Their channel model considers only pathloss and ignores the fading effects. In [19] and [20], Tsiftsis et.al. have considered $\mathrm{K}$ and Gamma-Gamma atmosphericinduced fading models without explicitly taking into account the path-loss and evaluated outage probability for a multi-hop FSO system. Their results demonstrate the usefulness of relayassisted transmission as a method to broaden the coverage area, but do not highlight its use as a fading-mitigation tool which is demonstrated in our paper.

In this paper, we study relay-assisted FSO communication system based on different configurations of relays whether they are employed in serial (i.e., multi-hop transmission) or in parallel (i.e., cooperative diversity). Our investigation on 
multi-hop FSO differs from earlier work in [18]-[20] in the sense that we explicitly take into account both path-loss and fading effects. At this point, it is worth emphasizing that fading variance is distance-dependent in FSO systems. This is a major difference between wireless RF and wireless optical systems and allows multi-hop FSO transmission bring performance improvements against the degrading effects of fading, as reflected by our performance analysis and numerical results. We also consider parallel relaying in this paper as a possible alternative to serial relaying. It is obvious that broadcast nature of wireless RF transmission (i.e., the cost-free possibility of the transmitted signals being received by other than destination nodes) is not present in FSO transmission which is based on line-of-sight transmission through directional beams. Therefore, we create an artificial broadcasting through the use of multiple transmitter apertures directed to relay nodes and propose a parallel relaying transmission scheme. For both parallel and serial transmission under consideration, we derive expressions for outage probability assuming amplifyand-forward (AF) and decode-and-forward (DF) relaying. We further present an extensive simulation study to confirm our derivations.

The rest of the paper is organized as follows: In Section II, we introduce the system model and describe the serial and parallel relay-assisted transmission schemes under consideration. In Section III, we present expressions of outage probability assuming both AF and DF relaying. In Section IV, we provide numerical results of outage analysis and compare them with simulation results. Finally, we conclude in Section V.

\section{RELAY-AsSisted FSO TRANSMisSion}

We consider a relay-assisted FSO communication system in which the transmitted signal from a source node propagates through $N$ serial or parallel relays before detection at the destination node. The system under consideration uses intensitymodulation direct-detection (IM/DD) employing binary pulse position modulation (BPPM). In such systems, optical transmitter is "on" during a half of the BPPM bit interval (i.e., "signal slot") and is "off" during the other half (i.e., "nonsignal slot"). The receiver integrates the detected photocurrent over both the signal and non-signal slots of the BPPM pulse and obtains the resulting electrical signal vector given by

$$
\mathbf{r}=\left[\begin{array}{c}
r^{s} \\
r^{n}
\end{array}\right]=\left[\begin{array}{c}
R T_{b}\left(P_{s}+P_{b}\right)+n^{s} \\
R T_{b} P_{b}+n^{n}
\end{array}\right]
$$

where $r^{s}$ and $r^{n}$ are the received electrical signals which correspond to signal and non-signal slots of the BPPM pulse. In (1), $P_{s}$ and $P_{b}$ are, respectively, the optical signal power and background power incident on the photodetector, $T_{b}$ is the duration of the signal and non-signal slots, and $R=\eta q / h f$ is responsitivity of the photodetector. $\eta$ is quantum efficiency of the photodetector, $q$ is the electron's charge, $h$ is Planck's constant, and $f$ is the optical frequency. $n^{s}$ and $n^{n}$ in (1) denote the additive noise terms for the signal and non-signal slots. We assume background noise limited receivers [4], [9] in which the shot noise caused by background radiation is dominant with respect to the other noise components, e.g., thermal, signal-dependent, and dark noise. Therefore, the noise term is modeled as a signal-independent additive white

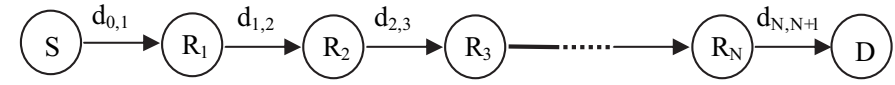

Fig. 1. FSO serial relaying configuration.

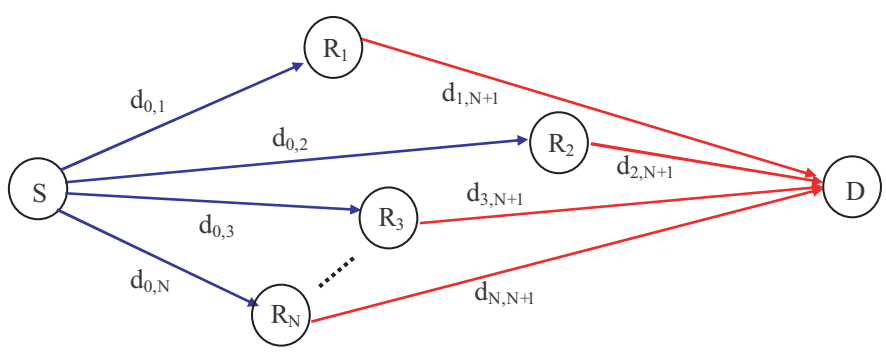

Fig. 2. FSO parallel relaying configuration.

Gaussian noise with zero mean and variance of $\sigma_{n}^{2}=N_{0} / 2$. This is a good approximation of the shot noise caused by background radiation when the intensity of the light incident on the photodetector is sufficiently high [2].

In serial relaying (Fig 1), the source transmits an intensitymodulated signal to a relay node. Under the assumption of DF relaying, the relay decodes the signal after direct detection, modulates it with BPPM, and retransmits it to the next relay. If AF relaying is employed, the relay does not perform any decoding on the received signal and, after multiplication with a proper energy scaling term, simply forwards it to the next relay. This continues until the source's data arrives at the destination node.

Fig. 2 illustrates a parallel relaying scheme. Since broadcasting is not possible due to the nature of FSO communication, the source is equipped with a multi-laser transmitter with each of the lasers pointing out in the direction of a corresponding relay node. The source node transmits the same signal to $N$ relays. Based on the AF or DF relaying method, the relays either decode and retransmit the signal or scale the received signal and forward it to the destination. It should be noted that, different from wireless RF communication, distributed spacetime block coding across relays is not required because of the ensured orthogonality of the received diffraction patterns from sufficiently separated transmit apertures [10], [21].

\section{A. Channel Model}

We consider an aggregated channel model where both distance-dependant path loss and turbulence-induced fading are taken into account. The path loss ratio for an FSO link with length $d$ can be expressed as [8]

$$
\ell=\frac{A_{T X} A_{R X}}{(\lambda d)^{2}} e^{-\sigma d}
$$

where $\sigma$ is the attenuation coefficient which is dependent on visibility [22]. $A_{T X}, A_{R X}$, and $\lambda$ are transmitter aperture area, receiver aperture area, and the optical wavelength, respectively.

We model turbulence-induced fading by log-normal distribution [4], [7]-[11] which is commonly used to model weak turbulence conditions. Let $\alpha=\exp (\chi)$ be the channel fading amplitude which is described by the log-normal probability 
distribution function (pdf) given by

$$
f(\alpha)=\frac{1}{\alpha \sqrt{2 \pi \sigma_{\chi}^{2}}} \exp \left(-\frac{\left(\ln (\alpha)-\mu_{\chi}\right)^{2}}{2 \sigma_{\chi}^{2}}\right) .
$$

Hence the fading log-amplitude $\chi$ is modeled as a Gaussian distributed random variable with mean $\mu_{\chi}$ and variance $\sigma_{\chi}^{2}$. We normalize the fading amplitude such that $E\left[\alpha^{2}\right]=1$ implying $\mu_{\chi}=-\sigma_{\chi}^{2}$. This ensures that the fading does not attenuate or amplify the average power [6]. Besides the link length $d$, the log-amplitude variance depends on wave number $(k)$, and refractive index structure constant $\left(C_{n}^{2}\right)$ and is given by

$$
\sigma_{\chi}^{2}(d)=0.124 k^{7 / 6} C_{n}^{2} d^{11 / 6}
$$

\section{B. DF Relaying}

In DF relaying, the relay decodes the signal after direct detection, modulates it using BPPM with optical power $P$, and retransmits it to the next relay (or to the destination in the last hop), only if the received signal-to-noise ratio (SNR) exceeds a given decoding threshold. Such a threshold is required to avoid the error propagation [13].

In serial relaying, the received signal at $i^{\text {th }}$ node $(i=$ $1,2, \ldots, N+1)^{1}$ is given by

$$
\mathbf{r}_{i}=\left[\begin{array}{c}
r_{i}^{s} \\
r_{i}^{n}
\end{array}\right]=\left[\begin{array}{c}
R T_{b}\left(P g_{i-1, i}+P_{b}\right)+n_{i}^{s} \\
R T_{b} P_{b}+n_{i}^{n}
\end{array}\right]
$$

where $r_{i}^{s}$ and $r_{i}^{n}$ are the received signals which correspond to signal and non-signal slots of the BPPM pulse. In (5), $g_{i-1, i}$ is the channel gain of the link connecting $(i-1)^{\text {th }}$ and $i^{\text {th }}$ nodes and $P$ is the average transmitted optical power per transmit aperture which is related to the total transmitted power $\left(P_{t}\right)$ by $P=P_{t} /(N+1)$ for serial relaying. It is obvious that the optical signal power incident on the photodetector is now given as $P_{s}=P g_{i-1, i}$. The channel gain of the link connecting $i^{\text {th }}$ and $j^{\text {th }}$ nodes $g_{i, j}(i, j=0,1, \ldots, N+1)$ can be formulated as

$$
g_{i, j}=\alpha_{i, j}^{2} L_{i, j}
$$

where $\alpha_{i, j}$ is the turbulence-induced fading amplitude and $L_{i, j}=\ell\left(d_{i, j}\right) / \ell\left(d_{0, N+1}\right)$ denotes the normalized path loss for the hop range between $i^{\text {th }}$ and $j^{\text {th }}$ nodes with respect to the distance of direct link between source and destination, i.e., $d_{0, N+1}$.

In parallel relaying, each of the relay nodes receives the transmitted signal from the corresponding transmit apertures pointed in their direction. The received signal at $i^{\text {th }}$ relay $(i=$ $1,2, \ldots, N)$ is given by

$$
\mathbf{r}_{i}=\left[\begin{array}{c}
r_{i}^{s} \\
r_{i}^{n}
\end{array}\right]=\left[\begin{array}{c}
R T_{b}\left(P g_{0, i}+P_{b}\right)+n_{i}^{s} \\
R T_{b} P_{b}+n_{i}^{n}
\end{array}\right]
$$

where the average optical power per transmit aperture $P$ can be obtained by dividing the total transmitted power by the number of the transmit apertures in parallel relaying configuration $^{2}$, i.e., $P=P_{t} /(2 N)$. The relay nodes decode their

\footnotetext{
${ }^{1}$ Throughout this paper, indexes $i=0$ and $i=N+1$ refer to the source and the destination nodes respectively, and indexes $i=1,2, \ldots, N$ refer to the relay nodes.

${ }^{2}$ Recall that the source is equipped with $N$ transmitters and each of $N$ relay nodes has one transmitter resulting in a total of $2 N$ transmit apertures for this scheme.
}

received signals after direct detection, modulate them with BPPM, and simultaneously retransmit to the destination. At the destination, we assume a large receiver field of view which allows all of the optical fields transmitted from different relay nodes are simultaneously detected. Let $\mathrm{D}$ denote the decoded set which is the set of relays having successfully decoded the signal (i.e., the received SNR exceeds the threshold). The received signal at the destination is superposition of the received optical powers transmitted from the decoded set [10], [21] and is given by

$$
\begin{aligned}
\mathbf{r}_{N+1} & =\left[\begin{array}{l}
r_{N+1}^{s} \\
r_{N+1}^{n}
\end{array}\right] \\
& =\left[\begin{array}{c}
R T_{b}\left(\sum_{i \in \mathrm{D}} P g_{i, N+1}+P_{b}\right)+n_{N+1}^{s} \\
R T_{b} P_{b}+n_{N+1}^{n}
\end{array}\right] .
\end{aligned}
$$

\section{AF Relaying}

In AF relaying, the relay first normalizes the received signal $\mathbf{r}_{\mathrm{i}}=\left[r_{i}^{s}, r_{i}^{n}\right]^{\mathrm{T}}$ by a factor of $E\left[r_{i}^{s}+r_{i}^{n}\right]$ to ensure the unity of average power. The relay then modulates the normalized signal by optical power $P$ and retransmits it to the next relay (or to the destination within the last hop). In effect, the average transmitted power from each relay remains constant as $P$. Furthermore, in order to improve power efficiency, the relay reduces the level of the received signal before normalization to remove the background light bias (i.e., $R T_{b} P_{b}$ ) which contains no information. However, reducing the signal level by $R T_{b} P_{b}$ may result in a negative signal. Therefore, this bias operation should be performed in such a way that ensures the nonnegativity of the signal which will be modulated.

Considering the bias and normalization operations, the received signal at $i^{\text {th }}$ node $(i=1,2, \ldots, N+1)$ for serial relaying is given as

$$
\begin{aligned}
\mathbf{r}_{i} & =\left[\begin{array}{c}
r_{i}^{s} \\
r_{i}^{n}
\end{array}\right]=\left[\begin{array}{c}
a_{i-1} g_{i-1, i} r_{i-1}^{s}+R T_{b} P_{b}-A_{i}^{\prime}+n_{i}^{s} \\
a_{i-1} g_{i-1, i} r_{i-1}^{n}+R T_{b} P_{b}-A_{i}^{\prime}+n_{i}^{n}
\end{array}\right] \\
& =\left[\begin{array}{c}
a_{i-1} g_{i-1, i} r_{i-1}^{s}+A_{i}+n_{i}^{s} \\
a_{i-1} g_{i-1, i} r_{i-1}^{n}+A_{i}+n_{i}^{n}
\end{array}\right]
\end{aligned}
$$

where $A_{i}=R T_{b} P_{b}-A_{i}^{\prime}=-\min \left\{a_{i-1} g_{i-1, i} r_{i-1}^{s}+\right.$ $\left.n_{i}^{s}, a_{i-1} g_{i-1, i} r_{i-1}^{n}+n_{i}^{n}\right\}$ is the $\mathrm{AF}$ bias term including the effects of background light bias $\left(R T_{b} P_{b}\right)$ and the bias operation $\left(-A_{i}^{\prime}\right)$ which leads to improvement of power efficiency. In practice, the relay node carries out this bias operation simply by decreasing the level of the received signals in the signal and non-signal BPPM slots until one of them reaches zero. Note that the bias operation does not cause any loss of signal information because the information is contained in the difference between the signal and non-signal slot levels which remains constant during the bias operation where the signal and non-signal slot levels are reduced by the same amount. In (9), the amplification factor at the $(i-1)^{\text {th }}$ node, $a_{i-1}$ $(i=2,3, \ldots, N+1)$, is defined as

$$
a_{i-1}=\frac{R T_{b} P}{\rho_{i-1}}
$$

where $\rho_{i-1}=E\left[r_{i-1}^{s}+r_{i-1}^{n}\right]$ is the normalization term. Note that for the signal transmitted from the source, normalization is obviously not performed, i.e., $a_{0}=R T_{b} P$. The details on 
the calculation of the normalization term can be found in the Appendix. The received signal at the destination is given by

$$
\begin{aligned}
& \mathbf{r}_{N+1}=\left[\begin{array}{c}
r_{N+1}^{s} \\
r_{N+1}^{n}
\end{array}\right] \\
& =\left[\begin{array}{c}
\prod_{i=0}^{N} a_{i} g_{i, i+1}+\sum_{j=1}^{N}\left(n_{j}^{s}+A_{j}\right) \prod_{i=j}^{N} a_{i} g_{i, i+1}+n_{N+1}^{s} \\
\sum_{j=1}^{N}\left(n_{j}^{n}+A_{j}\right) \prod_{i=j}^{N} a_{i} g_{i, i+1}+n_{N+1}^{n}
\end{array}\right] .
\end{aligned}
$$

In parallel relaying, the relays receive the transmitted signals from the corresponding transmit apertures pointed in the direction of their locations and simultaneously retransmit them with proper normalization and bias operations. The received signal at $i^{\text {th }}$ relay $(i=1,2, \ldots, N)$ is therefore given by

$$
\mathbf{r}_{i}=\left[\begin{array}{c}
r_{i}^{s} \\
r_{i}^{n}
\end{array}\right]=\left[\begin{array}{c}
R T_{b} P g_{0, i}+A_{i}+n_{i}^{s} \\
A_{i}+n_{i}^{n}
\end{array}\right]
$$

and the received signal at the destination is

$$
\begin{aligned}
& \mathbf{r}_{N+1}=\left[\begin{array}{c}
r_{N+1}^{s} \\
r_{N+1}^{n}
\end{array}\right] \\
& =\left[\begin{array}{c}
\sum_{i=1}^{N} a_{0} a_{i} g_{i, N+1} g_{0, i}+a_{i} g_{i, N+1}\left(n_{i}^{s}+A_{i}\right)+n_{N+1}^{s} \\
\sum_{i=1}^{N} a_{i} g_{i, N+1}\left(n_{i}^{n}+A_{i}\right)+n_{N+1}^{n}
\end{array}\right] .
\end{aligned}
$$

\section{OUtAge AnAlysis}

Atmospheric turbulence results in a very slowly-varying fading in FSO systems. The channel coherence time is about 1-100 ms, therefore fading remains constant over hundreds of thousand up to millions of consecutive bits for typical transmission rates [9]. For such quasi-static channels where the errors caused by fading are no longer independent, outage probability is a suitable metric to evaluate the system performance. Denote $C\left(\alpha^{\prime}\right)$ as the instantaneous capacity corresponding to a channel realization $\alpha=\alpha^{\prime}$ which is a function of instantaneous electrical SNR $\gamma$. For a Gaussian channel where the mean of received signal components for the signal and non-signal slots are given by $m^{s}$ and $m^{n}$, we have $r^{s} \sim N\left(m^{s}, \sigma_{n}^{2} / 2\right)$ and $r^{n} \sim N\left(m^{n}, \sigma_{n}^{2} / 2\right)$. Instantaneous electrical SNR can be then defined as [23]

$$
\gamma=\frac{\left(m^{s}-m^{n}\right)^{2}}{\sigma_{n}^{2}}
$$

The outage probability at the transmission rate of $R_{0}$ is given by [24]

$$
P_{\text {out }}\left(R_{0}\right)=\operatorname{Pr}\left(C(\gamma)<R_{0}\right) .
$$

Since $C(\cdot)$ is monotonically increasing with respect to $\gamma,(15)$ can be rewritten as

$$
P_{\text {out }}\left(R_{0}\right)=\operatorname{Pr}\left(\gamma<\gamma_{t h}\right)
$$

where $\gamma_{t h}=C^{-1}\left(R_{0}\right)$ is the threshold SNR. If SNR exceeds $\gamma_{t h}$, no outage happens and signal can be decoded with arbitrarily low error probability at the receiver. We also note that we assume the aforementioned decoding threshold in DF relaying and $\gamma_{t h}$ are taken equal.

\section{A. Outage Probability for DF Relaying}

In DF relaying, outage of each intermediate link may lead to the outage of the relaying scheme. Therefore, the calculation of outage probability for each intermediate link is required to evaluate the end-to-end performance. We first calculate the outage probability of an intermediate SISO (single-input single-output) link which is the building block of both serial and parallel relaying schemes.

Using (14), the received electrical SNR for the intermediate SISO link connecting $i^{\text {th }}$ and $j^{\text {th }}$ nodes can be obtained as

$$
\gamma=\frac{R^{2} T_{b}^{2} P^{2} g_{i, j}^{2}}{N_{0}}
$$

Inserting (17) in (16), the outage probability of the SISO link is

$$
P_{\text {out }, S I S O}=\operatorname{Pr}\left(g_{i, j}<\sqrt{\frac{\gamma_{t h} N_{0}}{R^{2} T_{b}^{2} P^{2}}}\right) .
$$

We replace the definition of $g_{i, j}=\alpha_{i, j}^{2} L_{i, j}$ in (18) and obtain

$$
P_{\text {out }, S I S O}\left(d_{i, j}\right)=\operatorname{Pr}\left(\alpha_{i, j}^{2}<\frac{1}{L_{i, j}} \frac{N^{\prime}}{P_{M}}\right)
$$

where $N^{\prime}=N+1$ for serial relaying and $N^{\prime}=2 N$ for parallel relaying. In (19), $P_{M}$ denotes power margin [9] and is defined as $P_{M}=P_{t} / P_{t h}$ where $P_{t h}$ denotes a threshold transmit power required to guarantee that no outage happens in a direct fading-free transmission from the source to the destination. Thus the power margin can be expressed as

$$
P_{M}=\sqrt{\frac{P_{t}^{2} R^{2} T_{b}^{2}}{N_{0} \gamma_{t h}}} .
$$

In (19), $\alpha_{i, j}^{2}$ is a log-normal random variable with mean $2 \mu_{\chi}\left(d_{i, j}\right)$ and variance $4 \sigma_{\chi}^{2}\left(d_{i, j}\right)$. Therefore the outage probability can be written using the cumulative distribution function (cdf) of the log-normal distribution as

$$
P_{\text {out }, \text { SISO }}\left(d_{i, j}\right)=Q\left(\frac{\ln \left(L_{i, j} P_{M} / N^{\prime}\right)+2 \mu_{\chi}\left(d_{i, j}\right)}{2 \sigma_{\chi}\left(d_{i, j}\right)}\right)
$$

where $Q(x)=(1 / \sqrt{2 \pi}) \int_{x}^{\infty} \exp \left(-u^{2} / 2\right) d u$. Once we obtain the outage probability of the SISO link, we can now return our attention to end-to-end outage probability for serial and parallel relaying.

1) Serial Relaying: In serial relaying, an outage occurs when any of the intermediate SISO links fails. Hence the outage probability for the end-to-end scheme can be given as

$$
P_{\text {out }}=\operatorname{Pr}\left(\bigcup_{i=0}^{N}\left\{\gamma_{i}<\gamma_{t h}\right\}\right)
$$

where $\gamma_{0}, \gamma_{1}, \ldots, \gamma_{N}$ are the SNRs of the intermediate SISO channels with the lengths of $d_{0,1}, d_{1,2}, \ldots, d_{N, N+1}$. Eq. (22) can be rewritten as

$$
\begin{aligned}
P_{\text {out }} & =1-\operatorname{Pr}\left(\bigcap_{i=0}^{N}\left\{\gamma_{i}>\gamma_{\text {th }}\right\}\right) \\
& =1-\prod_{i=0}^{N}\left(1-P_{\text {out }, \text { SISO }}\left(d_{i, i+1}\right)\right) .
\end{aligned}
$$


Replacing (21) in (23), the end-to-end outage probability for serial relaying scheme is obtained as

$P_{\text {out }}=1-\prod_{i=0}^{N}\left(1-Q\left(\frac{\ln \left(\frac{L_{i, i+1} P_{M} e^{2 \mu_{\chi}\left(d_{i, i+1}\right)}}{(N+1)}\right)}{2 \sigma_{\chi}\left(d_{i, i+1}\right)}\right)\right)$.

2) Parallel Relaying: In parallel relaying, outage occurrence in one of the intermediate SISO links does not necessarily lead to an outage of the relaying scheme. In this scheme, an outage occurs if either the decoded set D is empty or the MISO (multiple-input single-output) link between the decoding relays and the destination fails. The received SNR for the MISO link can be written as

$$
\gamma=\frac{R^{2} T_{b}^{2} P^{2}\left(\sum_{i \in \mathrm{D}} g_{i, N+1}\right)^{2}}{N_{0}} .
$$

Inserting (25) in (16), the outage probability of the MISO link is obtained as

$$
\begin{aligned}
P_{\text {out }, \text { MISO }} & =\operatorname{Pr}\left(\sum_{i \in \mathrm{D}} g_{i, N+1}<\sqrt{\frac{\gamma t h N_{0}}{R^{2} T_{b}^{2} P^{2}}}\right) \\
& =\operatorname{Pr}\left(\sum_{i \in \mathrm{D}} L_{i, N+1} \alpha_{i, N+1}^{2}<\frac{2 N}{P_{M}}\right) .
\end{aligned}
$$

We approximate the weighted sum of log-normal random variables as a log-normal random variable using moment matching method [11], [25], i.e., $\beta=\exp (\xi) \approx \sum_{i \in D} L_{i, N+1} \alpha_{i, N+1}^{2}$. The log-amplitude factor $\xi$ is defined as a normal random variable with mean $\mu_{\xi}$ and variance $\sigma_{\xi}^{2}$ which can be respectively written as

$$
\begin{gathered}
\mu_{\xi}\left(\bar{d}_{\mathrm{D}}\right)=\ln \sum_{i \in \mathrm{D}} L_{i, N+1}-\sigma_{\xi}^{2}\left(\bar{d}_{\mathrm{D}}\right) / 2 \\
\sigma_{\xi}^{2}\left(\bar{d}_{\mathrm{D}}\right)=\ln \left(1+\frac{\sum_{i \in \mathrm{D}} L_{i, N+1}^{2}\left(\mathrm{e}^{4 \sigma_{\chi}^{2}\left(d_{i, N+1}\right)}-1\right)}{\left(\sum_{i \in \mathrm{D}} L_{i, N+1}\right)^{2}}\right) .
\end{gathered}
$$

The mean and variance of the log-amplitude factor $\xi$ are functions of $\bar{d}_{\mathrm{D}}$ which is the set of all the distances between the decoding relays and the destination (i.e., $d_{i, N+1} \in \bar{d}_{\mathrm{D}}, \forall i \in \mathrm{D}$ ). Using the cdf of log-normal distribution, (26) is approximated as

$$
\begin{aligned}
P_{\text {out }, M I S O}\left(\bar{d}_{\mathrm{D}}\right) & \approx \operatorname{Pr}\left(\beta<\frac{2 N}{P_{M}}\right) \\
& =Q\left(\frac{\ln \left(P_{M} / 2 N\right)+\mu_{\xi}\left(\bar{d}_{\mathrm{D}}\right)}{\sigma_{\xi}\left(d_{\mathrm{D}}\right)}\right) .
\end{aligned}
$$

For a parallel relaying scheme with $N$ relays, the decoded set consists of $2^{N}$ possibilities. Let $\mathrm{S}(i)$ denote the $i^{\text {th }}$ possible set and $\operatorname{Pr}(\mathrm{S}(i))$ denote the probability of the event $\{\mathrm{D}=\mathrm{S}(i)\}$. The outage probability for parallel relaying scheme can be then expressed as

$$
P_{\text {out }}=\sum_{i=1}^{2^{N}} P_{\text {out }, M I S O}\left(\bar{d}_{\mathrm{S}(i)}\right) \operatorname{Pr}(\mathrm{S}(i)) .
$$

We can write $\operatorname{Pr}(\mathrm{S}(i))=\operatorname{Pr}\left[\left(\bigcap_{j \in \mathrm{S}(i)} j \in \mathrm{S}(i)\right) \cap\right.$ $\left.\left(\bigcap_{j \notin \mathrm{S}(i)} j \notin \mathrm{S}(i)\right)\right], \operatorname{Pr}(j \notin \mathrm{S}(i))=P_{\text {out }, S I S O}\left(d_{0, j}\right)$, and
$\operatorname{Pr}(j \in \mathrm{S}(i))=1-P_{\text {out }, S I S O}\left(d_{0, j}\right)$. Inserting these in (30), we obtain

$$
\begin{aligned}
P_{\text {out }}= & \sum_{i=1}^{2^{N}}\left(\prod_{j \in \mathrm{S}(i)}\left(1-P_{\text {out }, \text { SISO }}\left(d_{0, j}\right)\right)\right. \\
& \left.\times \prod_{j \notin \mathrm{S}(i)} P_{\text {out }, \text { SISO }}\left(d_{0, j}\right)\right) P_{\text {out }, M I S O}\left(\bar{d}_{\mathrm{S}(i)}\right) .
\end{aligned}
$$

Replacing (21) and (29) in (31), the end-to-end outage probability for parallel relaying scheme is obtained as

$$
\begin{aligned}
P_{\text {out }} \approx & \sum_{i=1}^{2^{N}}\left[\prod_{j \in \mathrm{S}(i)}\left(1-Q\left(\frac{\ln \left(\frac{L_{0, j} P_{M} e^{2 \mu_{\chi}}}{2 N}\right)}{2 \sigma_{\chi}\left(d_{0, j}\right)}\right)\right)\right. \\
& \left.\times \prod_{j \notin \mathrm{S}(i)} Q\left(\frac{\ln \left(\frac{L_{0, j} P_{M} e^{2 \mu_{\chi}}}{2 N}\right)}{2 \sigma_{\chi}\left(d_{0, j}\right)}\right)\right] Q\left(\frac{\ln \left(\frac{P_{M} e^{\mu_{\xi}}}{2 N}\right)}{\sigma_{\xi}\left(\bar{d}_{\mathrm{S}(i)}\right)}\right) .
\end{aligned}
$$

\section{B. Outage Probability for AF Relaying}

In AF relaying, the intermediate relay nodes forward the signal without any decoding. Thus, instead of considering SNRs in intermediate SISO links, the total received SNR at the destination should be calculated for outage analysis.

1) Serial AF Relaying: Recall that the received signal at the destination node for serial AF relaying is given by (11). Eq. (11) can be rewritten as

$$
\mathbf{r}_{N+1}=\left[\begin{array}{c}
r_{N+1}^{s} \\
r_{N+1}^{n}
\end{array}\right]=\left[\begin{array}{c}
\prod_{i=0}^{N} a_{i} g_{i, i+1}+A_{a c c}+n_{a c c}^{s} \\
A_{a c c}+n_{a c c}^{n}
\end{array}\right]
$$

where $n_{a c c}^{s}$ and $n_{a c c}^{n}$ are the accumulated noise terms which are defined as $n_{a c c}^{s}=\sum_{j=1}^{N} n_{j}^{s} \prod_{i=j}^{N} a_{i} g_{i, i+1}+n_{N+1}^{s}$, and $n_{a c c}^{n}=\sum_{j=1}^{N} n_{j}^{n} \prod_{i=j}^{N} a_{i} g_{i, i+1}+n_{N+1}^{n}$. In (33), $A_{a c c}$ denotes the accumulated bias term and is given by $A_{a c c}=$ $\sum_{j=1}^{N} A_{j} \prod_{i=j}^{N} a_{i} g_{i, i+1}$. Using (14) and (33), the received SNR at the destination node is given by

$$
\gamma=\frac{\prod_{i=0}^{N} a_{i}^{2} g_{i, i+1}^{2}}{N_{0}\left(\sum_{j=1}^{N} \prod_{i=j}^{N} a_{i}^{2} g_{i, i+1}^{2}+1\right)}
$$

Defining

$$
\nu_{j}=\frac{\prod_{i=0}^{j} a_{i}^{2} g_{i, i+1}^{2}}{\left(R T_{b}\right)^{2} P^{2}}
$$

we can rewrite (34) as

$$
\gamma=\frac{\left(R T_{b}\right)^{2} P^{2}\left(\nu_{0}^{-1}+\nu_{1}^{-1}+\cdots+\nu_{N}^{-1}\right)^{-1}}{N_{0}}
$$

where $\nu_{j}$ 's are log-normal random variables since any product of independent log-normal random variables is also lognormally distributed. Let $\nu_{j}=\exp \left(\kappa_{i}\right)$, then $\kappa_{i}$ is a normal random variable with mean

$$
\mu_{\kappa}(i)=\ln \left(L_{0,1}^{2} \prod_{j=1}^{i} a_{j}^{2} L_{j, j+1}^{2}\right)-4 \sum_{j=0}^{i} \sigma_{\chi}^{2}\left(d_{j, j+1}\right)
$$


and variance

$$
\sigma_{\kappa}^{2}(i)=16 \sum_{j=0}^{i} \sigma_{\chi}^{2}\left(d_{j, j+1}\right) .
$$

On the other hand, the covariance between $\kappa_{i}$ and $\kappa_{j}$ can be obtained as $\Sigma_{\kappa}(i, j)=\sigma_{\kappa}^{2}(\min (i, j))$. Since any power (positive or negative) of a log-normal random variable is also log-normally distributed, the sum of $\nu_{j}$ 's can be approximated as a $\log$-normal random variable, i.e., $\exp (\varepsilon) \approx \sum_{j=0}^{N} \nu_{j}^{-1}$. Therefore, $\gamma$ can be approximated in terms of a single lognormal random variable as

$$
\gamma \approx\left(R T_{b}\right)^{2} P^{2} \exp (-\varepsilon) / N_{0}
$$

where the mean and variance of the normally distributed random variable $\varepsilon$ are

$$
\begin{array}{r}
\mu_{\varepsilon}=\ln \left(\sum_{i=0}^{N} \exp \left(\sigma_{\kappa}^{2}(i) / 2-\mu_{\kappa}(i)\right)\right)-\sigma_{\varepsilon}^{2} / 2 \\
\sigma_{\varepsilon}^{2}=\ln \left(1+\frac{\sum_{i=0}^{N} \sum_{j=0}^{N} e^{\frac{\sigma_{\kappa}^{2}(i)+\sigma_{\kappa}^{2}(j)}{2}-\mu_{\kappa}(i)-\mu_{\kappa}(j)}\left(e^{\Sigma_{\kappa}(i, j)}-1\right)}{\left(\sum_{i=0}^{N} \exp \left(\sigma_{\kappa}^{2}(i) / 2-\mu_{\kappa}(i)\right)\right)^{2}}\right)
\end{array}
$$

Replacing (39) in (16), the end-to-end outage probability of the serial AF relaying scheme is obtained as

$$
\begin{aligned}
P_{\text {out }} & \approx \operatorname{Pr}\left(\exp (-\varepsilon)<\frac{\gamma_{t h} N_{0}}{\left(R T_{b}\right)^{2} P^{2}}\right) \\
& =\operatorname{Pr}\left(\exp (-\varepsilon)<\frac{(N+1)^{2}}{P_{M}^{2}}\right) \\
& =Q\left(\frac{\ln \left(P_{M}^{2} /(N+1)^{2}\right)-\mu_{\varepsilon}}{\sigma_{\varepsilon}}\right) .
\end{aligned}
$$

2) Parallel AF Relaying: Recall that the received signal at the destination node for parallel AF relaying is given by (13). Eq. (13) can be rewritten as

$$
\begin{aligned}
\mathbf{r}_{N+1} & =\left[\begin{array}{c}
r_{N+1}^{s} \\
r_{N+1}^{n}
\end{array}\right] \\
= & {\left[\begin{array}{c}
\sum_{i=1}^{N} P a_{i} g_{i, N+1} g_{0, i} x_{0}+A_{a c c}+n_{a c c}^{s} \\
A_{a c c}+n_{a c c}^{n}
\end{array}\right] }
\end{aligned}
$$

where we define the accumulated noise terms as $n_{a c c}^{s}=$ $\sum_{i=1}^{N} a_{i} g_{i, N+1} n_{i}^{s}+n_{N+1}^{s}, n_{a c c}^{n}=\sum_{i=1}^{N} a_{i} g_{i, N+1} n_{i}^{n}+n_{N+1}^{n}$, and the accumulated bias term as $A_{a c c}=\sum_{i=1}^{N} a_{i} g_{i, N+1} A_{i}$. Using (14) and (43), the received SNR at the destination node is given by

$$
\gamma=\frac{\left(R T_{b} P \sum_{i=1}^{N} a_{i} g_{i, N+1} g_{0, i}\right)^{2}}{N_{0}\left(\sum_{i=1}^{N} a_{i}^{2} g_{i, N+1}^{2}+1\right)}
$$

The summation terms in the numerator and denominator of (44) can be approximated as single log-normal random variables, i.e., $\exp \left(\omega_{1}\right) \approx \sum_{i=1}^{N} a_{i} g_{i, N+1} g_{0, i}$ and $\exp \left(\omega_{2}\right) \approx$ $\sum_{i=1}^{N} a_{i}^{2} g_{i, N+1}^{2}$. Therefore, (44) reduces to

$$
\gamma \approx \frac{R^{2} T_{b}^{2} P^{2} \exp \left(2 \omega_{1}\right)}{N_{0}\left(\exp \left(\omega_{2}\right)+1\right)}
$$

The log-amplitude pair $\left(\omega_{1}, \omega_{2}\right)$ follows a correlated bivariate normal distribution [26]. Their mean and covariance matrix are defined respectively as

$$
\begin{gathered}
\mu_{1}=\ln \sum_{i=1}^{N} a_{i} L_{0, i} L_{i, N+1}-\sigma_{1}^{2} / 2 \\
\mu_{2}=\ln \sum_{i=1}^{N} a_{i}^{2} L_{i, N+1}^{2} e^{4 \sigma_{\chi}^{2}\left(d_{i, N+1}\right)}-\sigma_{2}^{2} / 2 \\
\boldsymbol{\Sigma}=\left[\begin{array}{cc}
\sigma_{1}^{2} & \sigma_{12} \\
\sigma_{12} & \sigma_{2}^{2}
\end{array}\right]
\end{gathered}
$$

where $\sigma_{1}^{2}, \sigma_{2}^{2}$, and $\sigma_{12}$ are given by

$$
\begin{gathered}
\sigma_{1}^{2}=\ln \left(1+\frac{\sum_{i=1}^{N} a_{i}^{2} L_{0, i}^{2} L_{i, N^{\prime}}^{2}\left(e^{4\left(\sigma_{\chi}^{2}\left(d_{0 i}\right)+\sigma_{\chi}^{2}\left(d_{i N^{\prime}}\right)\right)}-1\right)}{\left(\sum_{i=1}^{N} a_{i} L_{0, i} L_{i, N^{\prime}}\right)^{2}}\right) \\
\left.\sigma_{2}^{2}=\ln \left(1+\frac{\sum_{i=1}^{N} a_{i}^{4} L_{i, N^{\prime}}^{4}\left(e^{24 \sigma_{\chi}^{2}\left(d_{i N^{\prime}}\right)}-e^{8 \sigma_{\chi}^{2}\left(d_{i N^{\prime}}\right)}\right)}{\left(\sum_{i=1}^{N} a_{i}^{2} L_{i, N^{\prime}}^{2} e^{4 \sigma_{\chi}^{2}\left(d_{i N^{\prime}}\right)}\right)^{2}}\right)^{2}\right)
\end{gathered}
$$

$\sigma_{12}=\ln \left(1+\frac{\sum_{i=1}^{N} a_{i}^{3} L_{0, i} L_{i, N^{\prime}}^{3}\left(e^{12 \sigma_{\chi}^{2}\left(d_{i N^{\prime}}\right)}-e^{4 \sigma_{\chi}^{2}\left(d_{i N^{\prime}}\right)}\right)}{\left(\sum_{i=1}^{N} a_{i}^{2} L_{i, N^{\prime}}^{2} e^{4 \sigma_{\chi}^{2}\left(d_{i N^{\prime}}\right)}\right)\left(\sum_{i=1}^{N} a_{i} L_{0, i} L_{i, N^{\prime}}\right)}\right)$

where $N^{\prime}=N+1$. Replacing (45) in (16), the end-to-end outage probability is obtained using pdf of the bivariate normal distribution as in (52) at the top of the next page. The upper limit of the inner integration in (52) is expressed as $\omega_{0}=$ $\ln \left(2 N\left(\exp \left(\omega_{2}\right)+1\right) / P_{M}\right) / 2$.

A closed-form expression for (52) is unfortunately not available. However, it can be easily calculated through multi-dimensional integration routines such as Gauss-Hermite quadrature formula [27].

\section{Numerical RESUlts AND Discussions}

In this section, we present numerical results for outage analysis obtained through the derived expressions and MonteCarlo simulations. In the following, we consider an FSO system with $\lambda=1550 \mathrm{~nm}$ operating in clear weather conditions with visibility of $10 \mathrm{~km}$. We assume an atmospheric attenuation of $0.43 \mathrm{~dB} / \mathrm{km}$ (i.e., $\sigma \approx 0.1$ ) and structure constant of $C_{n}^{2}=1 \times 10^{-14} \mathrm{~m}^{-2 / 3}$. The link range (i.e., distance between the source and the destination) is $d_{0, N+1}=5 \mathrm{~km}$. For serial relaying, we assume the consecutive nodes are equidistant along the path from the source to the destination. In parallel relaying, the relays are located on the halfway point.

Fig. 3 demonstrates the end-to-end outage probability of an FSO DF system for serial relaying assuming $N=1,2,3$. We present analytical results which have been obtained through (24) along with the Monte-Carlo simulation of (16). As clearly seen from Fig. 3, our exact closed-form expressions provide an identical match to simulation results. As a benchmark, outage probability of the direct transmission is also included in this 


$$
\begin{aligned}
P_{\text {out }} & =\operatorname{Pr}\left(\frac{\exp \left(2 \omega_{1}\right)}{\exp \left(\omega_{2}\right)+1}<\frac{2 N}{P_{M}}\right) \\
& =\int_{-\infty}^{\infty} \int_{-\infty}^{\omega_{0}} \frac{1}{2 \pi \sqrt{|\boldsymbol{\Sigma}|}} \exp \left(-\frac{\left(\sigma_{2}^{2}\left(\omega_{1}-\mu_{1}\right)^{2}+\sigma_{1}^{2}\left(\omega_{2}-\mu_{2}\right)^{2}-2 \sigma_{12}\left(\omega_{1}-\mu_{1}\right)\left(\omega_{2}-\mu_{2}\right)\right)}{2|\boldsymbol{\Sigma}|}\right) d \omega_{2} d \omega_{1}
\end{aligned}
$$

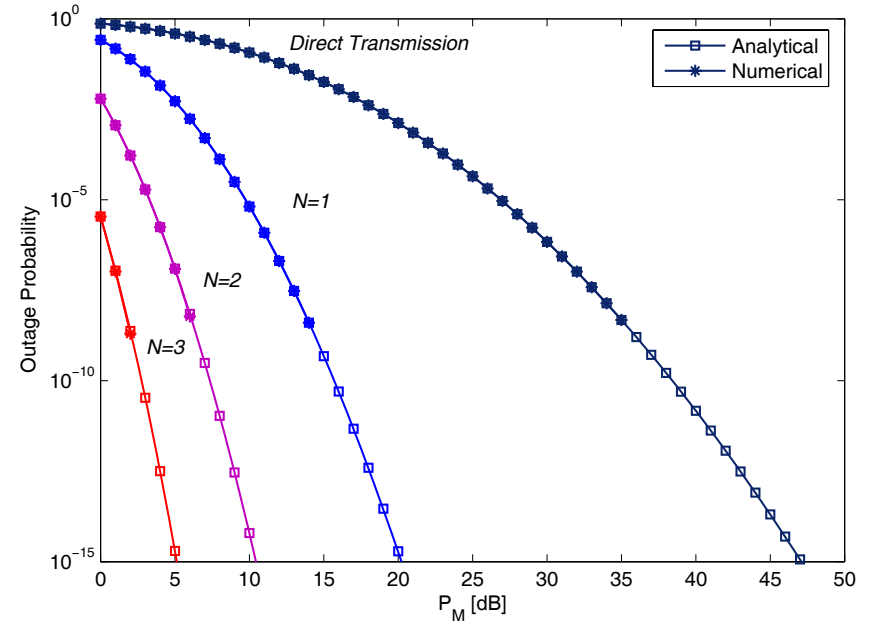

Fig. 3. Outage probability of FSO serial decode-and-forward relaying scheme.

figure. Serial relaying significantly improves the performance. Particularly, for a target outage probability of $10^{-6}$, we observe performance improvements of $18.5 \mathrm{~dB}, 25.4 \mathrm{~dB}$, and $29.2 \mathrm{~dB}$ for $N=1,2$, and 3 with respect to the direct transmission. We should emphasize that these impressive performance gains are a result of relay-assisted transmission's ability to exploit the distance-dependency of the log-amplitude variance. As reported in [11], spatial diversity, through the use of colocated apertures, in an FSO communication system scales down the effective log-amplitude variance by the number of apertures. A similar effect is observed in serial relaying where the fading log-amplitude variance of each intermediate SISO link decreases as the intra-distance decreases through the insertion of relaying nodes. Therefore, diversity advantage is artificially induced in the relaying scheme by shortening the distance between communicating nodes.

Fig. 4 demonstrates the end-to-end outage probability of an FSO DF system for parallel relaying assuming $N=1,2,3$. We present analytical results which have been obtained through (32) along with the Monte-Carlo simulation of (16). Although the derived expression is based upon an approximation, we have found nearly identical match between analytical and simulation results. We also note that for $N=1$ the performance of serial and parallel relaying coincide as expected which can be readily confirmed through the comparison of (24) and (32). We observe from Fig. 4 that parallel relaying improves the outage performance with respect to the direct transmission. Specifically, we obtain performance improvements of $18.5 \mathrm{~dB}$, $20.3 \mathrm{~dB}$, and $20.7 \mathrm{~dB}$ for $N=1,2$ and 3 with respect to the direct transmission for a target outage probability of $10^{-6}$. We note that performance gains are less than those observed in serial relaying since parallel relaying (with only two hops)

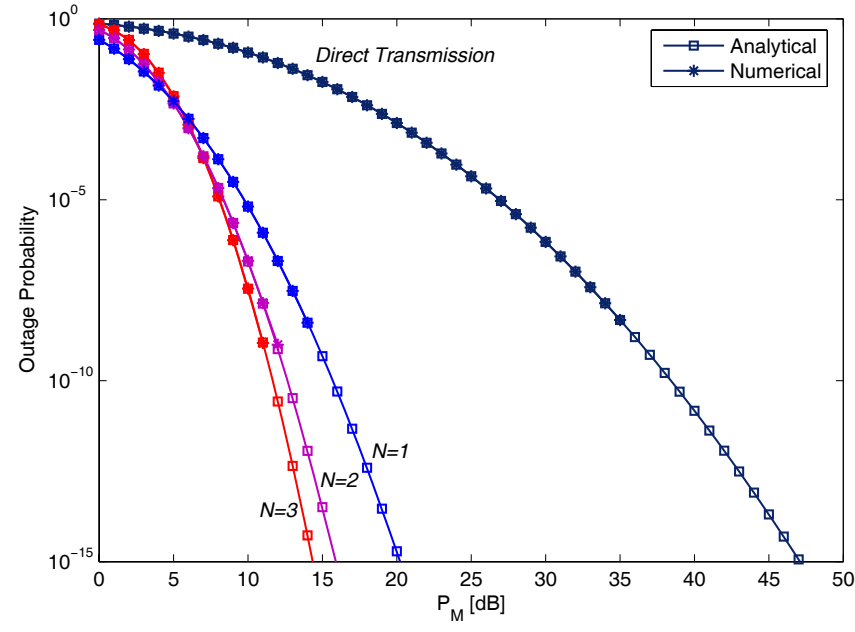

Fig. 4. Outage probability of FSO parallel decode-and-forward relaying scheme.

exploits distance-dependency of fading variance to a lesser extent.

Fig. 5 demonstrates the end-to-end outage probability of an FSO AF system for serial relaying assuming $N=1,2,3$. We present approximate analytical results which have been obtained through (42) along with the Monte-Carlo simulation of (16). For $N=1$, we observe a very good match between analytical and simulation results. The discrepancy gets larger for $N=2$ and $N=3$. Recall that closed-form expressions for this case are built upon an approximation of the sum of correlated log-normal random variables as a single lognormal term. Comparison with direct transmission reveals that performance improvements are $12.2 \mathrm{~dB}, 17.7 \mathrm{~dB}$, and $21 \mathrm{~dB}$ for $N=1,2$ and 3 for a target outage probability of $10^{-6}$. It is observed that the performance gains are less than those observed in DF relaying. However, AF relays enjoy a lower complexity in comparison with DF counterparts since it does not require any decoding process.

Fig. 6 demonstrates the end-to-end outage probability of an FSO AF system for parallel relaying assuming $N=1,2,3$. We present analytical results which have been obtained through (52) along with the Monte-Carlo simulation of (16). Similar to Fig. 5, they provide a good match for $N=1$ while some discrepancy is observed for $N=2$, and 3 due to the lognormal approximation. At a target outage probability of $10^{-6}$, FSO DF parallel relaying system yields performance gains of $12.2 \mathrm{~dB}, 18.1 \mathrm{~dB}$, and $20.2 \mathrm{~dB}$ for $N=1,2$, and 3 . It is observed that these are lower than those observed for its counterpart with DF relaying.

\section{Conclusions}

In this paper, we have applied the concept of relaying to FSO systems. We have investigated serial and parallel 


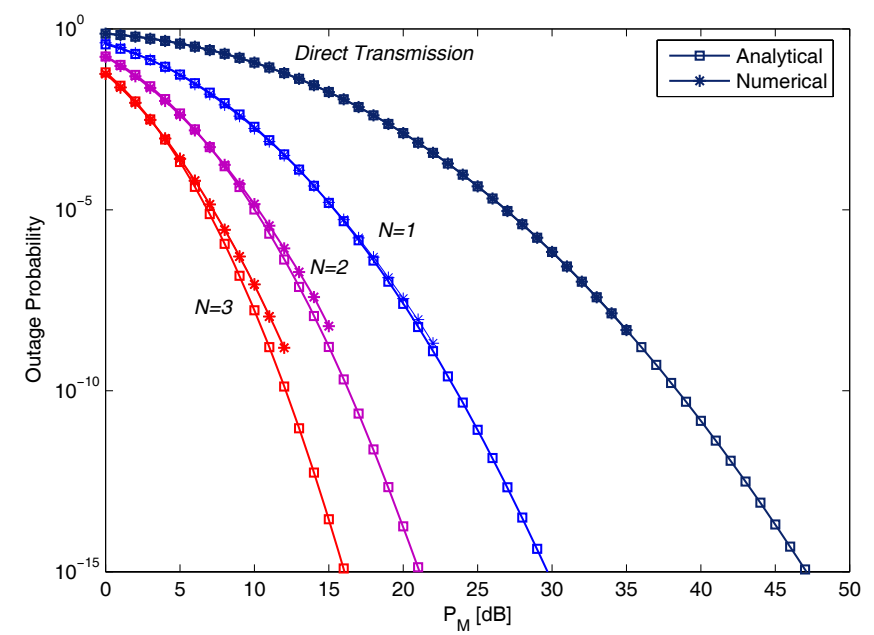

Fig. 5. Outage probability of FSO serial amplify-and-forward relaying scheme.

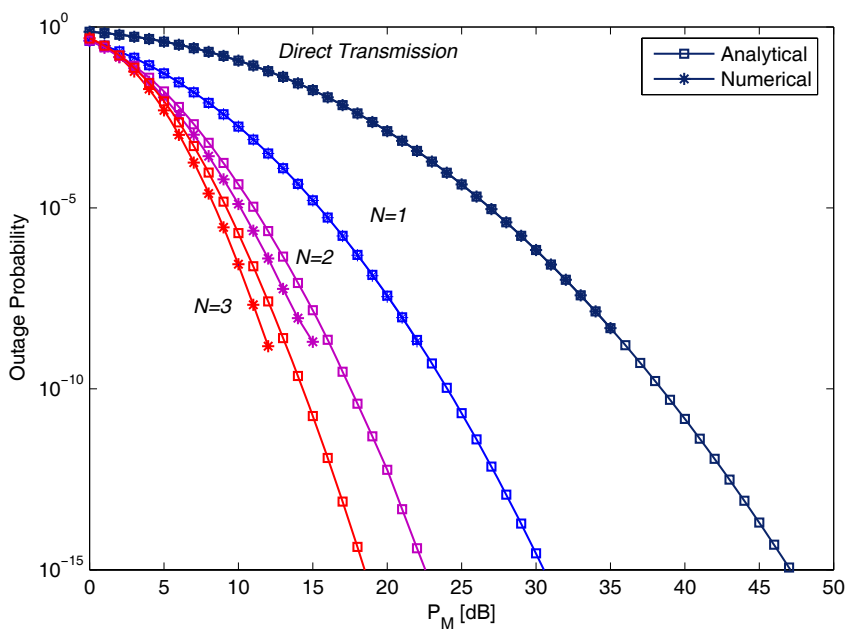

Fig. 6. Outage probability of FSO parallel amplify-and-forward relaying scheme.

relaying, each of which operates either in AF or DF modes. The fact that fading variance is distance-dependent in FSO systems constitutes a major difference between wireless RF and wireless optical systems. This lets multi-hop FSO transmission smartly exploit the shorter distance in the resulting hops and brings substantial improvements against the degrading effects of turbulence-induced fading. As a possible alternative to serial relaying, we have also considered parallel relaying where an artificial broadcasting is obtained through the use of multiple transmitter apertures directed to relay nodes. Through the derivation of outage probability and Monte-Carlo simulations, we have quantified the performance improvements obtained for both parallel and serial relaying schemes. Specifically, for a single-relay serial relaying scheme (i.e., double-hop scheme), performance improvements of $12.2 \mathrm{~dB}$ and 18.5 $\mathrm{dB}$ have been obtained for serial relaying with $\mathrm{AF}$ and $\mathrm{DF}$ modes. For a triple-hop scheme, the respective performance improvements climb up to $17.7 \mathrm{~dB}$ and $25.4 \mathrm{~dB}$. In comparison to serial relaying, parallel relaying takes advantage of distancedependency of fading log-normal variance to a lesser extent and is outperformed by its competitor as the number of relays increases.

\section{APPENDIX}

\section{CALCUlation OF THE NORMaLization TERM IN AF} RELAYING

The normalization term at $i^{\text {th }}$ relay $(i=1,2, \ldots, N)$ is given by

$$
\rho_{i}=E\left[r_{i}^{s}+r_{i}^{n}\right] .
$$

After the insertion of the bias term, either $r_{i}^{s}$ or $r_{i}^{n}$ becomes zero. Therefore, we can rewrite the normalization term as

$$
\rho_{i}=E\left[\left|r_{i}^{s}-r_{i}^{n}\right|\right] \text {. }
$$

Conditioned on $\bar{g}_{i}=\left\{g_{0,1}, g_{1,2} \ldots, g_{i-1, i}\right\}$, the term $r_{i}^{s}-r_{i}^{n}$ is real Gaussian with mean $m_{i}=\prod_{j=0}^{i-1} a_{j} g_{j, j+1}$ and variance $s_{i}^{2}=\left(1+\sum_{j=1}^{i-1} \prod_{k=j}^{i-1} a_{k} g_{k, k+1}\right) N_{0}$ for serial AF relaying and with mean $m_{i}=R T_{b} P g_{0, i}$ and variance $s_{i}^{2}=N_{0}$ for parallel AF relaying. Its magnitude, therefore, follows a folded-normal distribution [28]. Hence, the normalization term conditioned on $\bar{g}_{i}$ is given by [28]

$$
\begin{aligned}
E\left[r_{i}^{s}+r_{i}^{n} \mid \bar{g}_{i}\right]= & s_{i}\left(\bar{g}_{i}\right) \sqrt{\frac{2}{\pi}} \exp \left(-\frac{m_{i}^{2}\left(\bar{g}_{i}\right)}{2 s_{i}^{2}\left(\bar{g}_{i}\right)}\right) \\
& +m_{i}\left(\bar{g}_{i}\right)\left(2 Q\left(-\frac{m_{i}\left(\bar{g}_{i}\right)}{s_{i}\left(\bar{g}_{i}\right)}\right)-1\right) .
\end{aligned}
$$

Performing an expectation over $\bar{g}_{i}$, we obtain

$$
\begin{aligned}
\rho_{i}=\int_{\bar{g}_{i}} f_{\bar{g}_{i}}\left(\bar{g}_{i}\right)[ & s_{i}\left(\bar{g}_{i}\right) \sqrt{\frac{2}{\pi}} \exp \left(-\frac{m_{i}^{2}\left(\bar{g}_{i}\right)}{2 s_{i}^{2}\left(\bar{g}_{i}\right)}\right) \\
& \left.+m_{i}\left(\bar{g}_{i}\right)\left(2 Q\left(-\frac{m_{i}\left(\bar{g}_{i}\right)}{s_{i}\left(\bar{g}_{i}\right)}\right)-1\right)\right] d \bar{g}_{i}
\end{aligned}
$$

where $f_{\bar{g}_{i}}\left(\bar{g}_{i}\right)$ is the joint pdf of the log-normal vector $\bar{g}_{i}$.

The calculation of (56) requires numerical computation, but it can be also precisely approximated which can be useful particularly for practical implementation. The normalization term can be rewritten by the law of total probability as

$$
\begin{aligned}
\rho_{i} & =E\left[\left|r_{i}^{s}-r_{i}^{n}\right|\right] \\
& =\operatorname{Pr}\left(r_{i}^{s}>r_{i}^{n}\right) E\left[r_{i}^{s}-r_{i}^{n}\right]+\operatorname{Pr}\left(r_{i}^{n}>r_{i}^{s}\right) E\left[r_{i}^{n}-r_{i}^{s}\right] .
\end{aligned}
$$

Let $P_{e}$ denote the average error probability for BPPM modulation scheme. We can then write $\operatorname{Pr}\left(r_{i}^{s}>r_{i}^{n}\right)=1-P_{e} \approx 1$ and $\operatorname{Pr}\left(r_{i}^{n}>r_{i}^{s}\right)=P_{e}<<1$. Inserting these in (57), the normalization term can be approximated as

$$
\begin{aligned}
\rho_{i} & =E\left[\left|r_{i}^{s}-r_{i}^{n}\right|\right] \approx E\left[r_{i}^{s}-r_{i}^{n}\right]=E\left[m_{i}\right] \\
& =\left\{\begin{array}{lr}
\prod_{j=0}^{i-1} a_{j} L_{j, j+1} & \text { serial relaying } \\
R T_{b} P L_{0, i} & \text { parallel relaying }
\end{array}\right.
\end{aligned}
$$

Inserting (58) in (10), the amplification factor is obtained as

$$
a_{i}=\left\{\begin{array}{lr}
\frac{R T_{b} P}{\prod_{j=0}^{i-1} a_{j} L_{j, j+1}}=\frac{1}{L_{i-1, i}} & \text { serial relaying } \\
\frac{R T_{b} P}{R T_{b} P L_{0, i}}=\frac{1}{L_{0, i}} & \text { parallel relaying }
\end{array}\right.
$$

Inserting (59) in (58), the normalization term reduces to

$$
\rho_{i} \cong\left\{\begin{array}{l}
R T_{b} P L_{i-1, i} \quad \text { serial relaying } \\
R T_{b} P L_{0, i} \quad \text { parallel relaying }
\end{array}\right.
$$

It is obvious that the approximated normalization terms in (60) are very simple in comparison to (56) and can be easily implemented at the relay terminals. Our simulation results further indicate that this approximation results in negligible difference within the line of thickness. 


\section{REFERENCES}

[1] R. G. Hunsperger, Photonic Devices and Systems. New York: Marcel Dekker, 1994.

[2] L. C. Andrews, R. L. Phillips, and C. Y. Hopen, Laser Beam Scintillation with Applications. SPIE Press, 2001.

[3] D. Kedar and S. Arnon, "Urban optical wireless communication networks: the main challenges and possible solutions," IEEE Commun. Mag., vol. 42, no. 5, pp. s2-s7, May 2004.

[4] X. Zhu and J. M. Kahn, "Free-space optical communication through atmospheric turbulence channels," IEEE Trans. Commun., vol. 50, no. 8, pp. 1293-1300, Aug. 2002.

[5] X. Zhu and J. M. Kahn, "Performance bounds for coded free-space optical communications through atmospheric turbulence channels," IEEE Trans. Commun., vol. 51, no.8, p. 1233-1239, Aug. 2003.

[6] M. Uysal, S. M. Navidpour, and J. Li, "Error rate performance of coded free-space optical links over strong turbulence channels," IEEE Commun. Lett., vol. 8, no. 10, p.635-637, Oct. 2004.

[7] X. Zhu and J. M. Kahn, "Markov chain model in maximum-likelihood sequence detection for free-space optical communication through atmospheric turbulence channels," IEEE Trans. Commun., vol. 51, no. 3, p. 509-516, Mar. 2003.

[8] S. M. Haas, "Capacity of and coding for multiple-aperture wireless optical communications, Ph.D. dissertation, Massachusetts Institute of Technology, 2003.

[9] E. Lee and V. Chan, "Part 1: optical communication over the clear turbulent atmospheric channel using diversity," IEEE J. Select. Areas Commun., vol. 22, no. 9, pp. 1896-1906, 2004.

[10] S. G. Wilson, M. Brandt-Pearce, Q. Cao, and M. Baedke, "Optical repetition MIMO transmission with multipulse PPM," IEEE J. Select. Areas Commun., vol. 23, no. 9, p. 1901-1909, Sept. 2005.

[11] S. M. Navidpour, M. Uysal, and M. Kavehrad, "BER performance of free-space optical transmission with spatial diversity," IEEE Trans. Wireless Commun., vol. 6, no. 8, p. 2813-2819, Aug. 2007

[12] J. N. Laneman and G. W. Wornell, "Energy-efficient antenna sharing and relaying for wireless networks," in Proc. IEEE Wireless Communications and Networking Conf. (WCNC), Chicago, IL, Sept. 2000.

[13] J. N. Laneman, D. N. C. Tse, and G. W. Wornell, "Cooperative diversity in wireless networks: efficient protocols and outage behavior," IEEE Trans. Inform. Theory, vol. 50, no. 12, pp. 3062-3080, Dec. 2004.

[14] A. Sendonaris, E. Erkip, and B. Aazhang, "User cooperation diversitypart I: system description," IEEE Trans. Commun., vol. 51, pp. 19271938, Nov. 2003.

[15] M. O. Hasna and M. S. Alouini, "Outage probability of multihop transmission over Nakagami fading channels," IEEE Commun. Lett., vol. 7, pp. 216-218, May 2003.

[16] G. K. Karagiannidis, T. A. Tsiftsis, and R. K. Mallik, "Bounds for multihop relayed communications in Nakagami-m fading," IEEE Trans. Commun., vol. 54, pp. 18-22, Jan. 2006.

[17] A. S. Acampora and S. V. Krishnamurthy, "A broadband wireless access network based on mesh-connected free-space optical links," IEEE Personal Commun., vol. 6, pp. 62-65, Oct. 1999.

[18] J. Akella, M. Yuksel, and S. Kalyanaraman, "Error analysis of multihop free-space optical communication," in Proc. IEEE International Conference on Communications 2005 (ICC'05), Seoul, Korea, May 2005.

[19] T. A. Tsiftsis, H. G. Sandalidis, G. K. Karagiannidis, and N. C. Sagias, "Multihop free-space optical communications over strong turbulence channels," in Proc. IEEE International Conference on Communications 2006 (ICC'06), Istanbul, Turkey, June 2006.

[20] G. K. Karagiannidis, T. A. Tsiftsis, H. G. Sandalidis, "Outage probability of relayed free space optical communication systems," Electron. Lett., vol. 42, pp. 994-995, Aug. 2006.
[21] M. Safari and M. Uysal, "Do we really need OSTBC for free-space optical communication with direct detection?" IEEE Trans. Wireless Commun., vol. 7, no. 11, Nov. 2008.

[22] I. I. Kim, J. Koontz, H. Hakakha, P. Adhikari, R. Stieger, C. Moursund, M. Bar-clay, A. Stanford, R. Ruigrok, J. Schuster, and E. Korevaar, "Measurement of scintillation and link margin for the TerraLink laser communication system," in Proc. SPIE, 3232, pp. 100-118, 1998.

[23] S. Dolinar, D. Divsalar, J. Hamkins, and F. Pollara, "Capacity of pulseposition modulation (PPM) on Gaussian and Webb channels," JPL TMO Progress Report, vol. 42-142, pp. 1-31, Apr.-June 2000.

[24] E. Biglieri, J. Proakis, and S. Shamai, "Fading channels: information theoretic and communications aspects," IEEE Trans. Inform. Theory, vol. 44, no. 6, pp. 2619-2692, Oct. 1998.

[25] L. F. Fenton, "The sum of lognormal probability distributions in scatter transmission systems," IRE Trans. Commun., vol. COM-8, pp. 57-67, Mar. 1960.

[26] M. Evans, N. Hastings, and B. Peacock, Statistical Distributions, 3rd edition. John Wiley \& Sons, 2000.

[27] M. K. Simon and M. S. Alouini, Digital Communication over Fading Channels. John Wiley \& Sons, 2000.

[28] F. C. Leone, L. S. Nelson, and R. B. Nottingham, "The folded normal distribution," Technometrics, vol. 3, pp. 543-550, 1961.

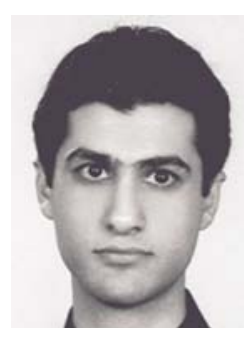

Majid Safari (S'08) received B.Sc. in Electrical Engineering from the University of Tehran, Tehran, Iran, in 2003 and M.S. in Electrical Engineering from Sharif University of Technology, Tehran, Iran, in 2005. He is currently a Ph.D. student in Electrical Engineering, University of Waterloo, Canada. His research interests include cooperative communications, free-space optical communications, and optical fiber communications.

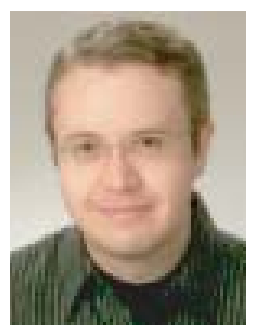

Murat Uysal was born in Istanbul, Turkey in 1973. $\mathrm{He}$ received the B.Sc. and the M.Sc. degree in electronics and communication engineering from Istanbul Technical University, Istanbul, Turkey, in 1995 and 1998, respectively, and the Ph.D. degree in electrical engineering from Texas A\&M University, College Station, Texas, in 2001. Since 2002, he has been with the Department of Electrical and Computer Engineering, University of Waterloo, Canada, where he is now an Associate Professor. His general research interests lie in communications theory and signal processing for communications with special emphasis on wireless applications. Specific research areas include space-time coding, MIMO techniques, performance analysis over fading channels, and cooperative communications.

Dr. Uysal is an Associate Editor for IEEE TRANS ACTIONS ON WIRELESS COMMUNICATIONS and IEEE COMMUNICATIONS LETTERS. He also served as a Guest Co-Editor for Wiley JOURNAL ON WIRELESS COMMUNICATIONS And Mobile Computing's special issue on "MIMO Communications" published in 2004. Over the years, he has served on the technical program committee of more than 50 international conferences in the communications area. He recently co-chaired IEEE ICC'07 Communication Theory Symposium. He is currently chairing Communications and Networking Symposium within the Canadian Conference on Electrical and Computer Engineering. Dr. Uysal is a Senior IEEE member. 